\title{
A!
}

This is an electronic reprint of the original article.

This reprint may differ from the original in pagination and typographic detail.

Tayyab, Muhammad; Koudouridis, G. P.; Gelaberr, X.; Jäntti, Riku

\section{Receiver Power Consumption during Handover in LTE}

Published in:

2019 IEEE 2nd 5G World Forum (5GWF)

DOI:

10.1109/5GWF.2019.8911641

Published: 01/01/2019

Document Version

Peer reviewed version

Please cite the original version:

Tayyab, M., Koudouridis, G. P., Gelaberr, X., \& Jäntti, R. (2019). Receiver Power Consumption during Handover in LTE. In 2019 IEEE 2nd 5G World Forum (5GWF) (pp. 74-79). IEEE.

https://doi.org/10.1109/5GWF.2019.8911641

This material is protected by copyright and other intellectual property rights, and duplication or sale of all or part of any of the repository collections is not permitted, except that material may be duplicated by you for your research use or educational purposes in electronic or print form. You must obtain permission for any other use. Electronic or print copies may not be offered, whether for sale or otherwise to anyone who is not an authorised user. 


\section{Receiver Power Consumption during Handover in LTE}

\author{
M. Tayyab ${ }^{1,3}$ \\ ${ }^{1}$ Huawei Technologies Finland Oy \\ Helsinki, Finland \\ muhammad.tayyab5@huawei.com \\ muhammad.tayyab@aalto.fi
}

\author{
G. P. Koudouridis ${ }^{2}$ and X. Gelabert ${ }^{2}$ \\ ${ }^{2}$ Huawei Technologies Sweden AB, \\ Kista, Sweden \\ george.koudouridis@huawei.com, \\ xavier.gelabert@huawei.com
}

\author{
R. Jäntti ${ }^{3}$ \\ ${ }^{3}$ Department of Communications and \\ Networking, School of Electrical \\ Engineering, Aalto University, Espoo, \\ Finland \\ riku.jantti@aalto.fi
}

\begin{abstract}
Recently, handover (HO) has gathered huge interest as the cellular mobile users desire for better quality of service (QoS) and continuous connection has increased. The power consumption is increasing day by day due to growing data rate demands of the users that directly impacts operators' operational expenditures (OPEX) and, not least, the environment by increased $\mathrm{CO}_{2}$ emissions. In this paper, we address the power consumption caused by the air-interface signaling messages received at both the eNB and User Equipment (UE) during $\mathrm{HO}$ in a Long Term Evolution (LTE) cellular network. A receiver power consumption model is presented with a detailed quantitative analysis using system level simulations. Numerical results indicate that the largest contributor to received air-interface HO signaling overhead is the reception of the measurement report by the eNB.
\end{abstract}

Keywords-LTE, handover, simulation, received power consumption, performance evaluation.

\section{INTRODUCTION}

In high data traffic areas, the cellular operators usually choose to place more base stations (BSs) to increase the capacity of the network by shrinking the coverage of eNBs and consequently improving the frequency reuse. In addition to supporting high data rate applications, cellular networks will need to provide their services to mobile users, probably at high speeds, within vehicles. The most challenging requirement for a wireless network to be ubiquitous is its ability to permit mobility without loss of connectivity and quality of service (QoS) degradation. But a limiting factor in densification planning is the handover $(\mathrm{HO})$ rate. The $\mathrm{HO}$ decision is a crucial component in the design process of $\mathrm{HO}$ since the efficiency and the success depend on the timeliness and accuracy of the decision. An increased $\mathrm{HO}$ rate due to high cell density counter-balances the gains in capacity, since the HO procedure signaling overheads reduce the user throughput [1]. A high density of the BSs may also result in increased power consumption due to unnecessary HOs with ping-pong events, frequent HOs and higher chances of $\mathrm{HO}$ failures (HOF). Altogether, this causes a more detrimental effect on the user device battery lifetime.

With the above in mind, in this work we will study the average supply power consumption that results from the reception of HO-related signaling messages over the air-interface in a Long Term Evolution (LTE) cellular network. The impact of

This project has received funding from the European Union's H2020 research and innovation program under grant agreement H2020-MCSA-ITN2016-SECRET 722424 [16]. user speed, cell densification and $\mathrm{HO}$ decision related parameters is studied with a breakdown of the different signaling reception contributions. In this paper we are specifically interested in the power consumption of the signaling messages received over the air-interface, to identify those individual processes of the $\mathrm{HO}$ procedure that are more power consuming.

It is worthy to mention that many eNB power consumption models exist in the literature, whereas very few works, in comparison, are devoted to LTE handset models. An eNB power consumption model by considering its main power consuming elements was proposed in [2][3], whereas an LTE handset power consumption model was proposed in [4].

In our previous work [5], we showed the impact of varying the inter-site distance (ISD), User Equipment (UE) speed and $\mathrm{HO}$ related parameters on the HO performance parameters and we found that increasing/decreasing the cell size, after a certain limit, degrades the HO performance mainly due to the uplink (UL) limitations. In [6], we analyzed the signaling overheads and the power consumption during the transmission of $\mathrm{HO}$ related signaling messages. We found that the measurement report (MeasReport) transmission is the major contributor to the airinterface signaling. We also noticed that the random access channel (RACH) signaling overhead transmission is the main power consuming part due to higher utilization of the resources (i.e. resource blocks). The work presented herein complements our previous work by addressing the received power consumption model (for reception of the signaling message over the air-interface) and the calculation of the transport blocks (TBs) used for each signaling message according to the 3GPP specified standards. To the best of our knowledge, this is the first work that shows the study of received power consumption of the $\mathrm{HO}$ procedure.

The rest of the paper is organized as follows: Section II provides an overview of the HO mechanism in LTE. Section III discusses the simulator modeling aspects and the power consumption model. In Section IV, numerical results are presented. And finally, Section V provides conclusion with future directions.

\section{OVERVIEW OF HANDOVER MECHANISM IN LTE}

The overall HO procedure in an LTE network is synthesized in Fig. 1 [7]. At large, the procedure can be divided into four differentiated phases: measurement, $\mathrm{HO}$ preparation, HO execution and $\mathrm{HO}$ completion phase. 


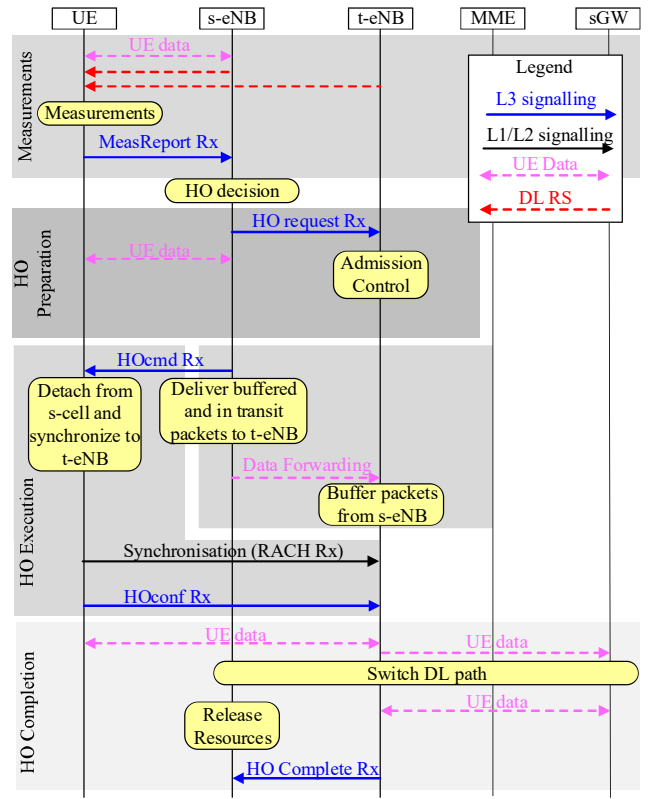

Fig. 1. HO procedure (adapted from [7]) considering no change of Mobility Management Entity (MME) and no change of serving gateway (sGW), also known as intra-MME/SG.

The measurement phase starts with the measurement of downlink (DL) reference signals (RS) from both the serving eNB (s-eNB) and neighboring eNBs, including the potential target eNB (t-eNB), which is the eNB "taking over" the handovered UE. The UE carries out signal strength (SS) measurements over a set of specific RS sent by the s-cell as well as the neighboring cells, and it computes the RS received power (RSRP) from each cell. After processing the measurements, if an "entry condition" is fulfilled, a measurement report (MeasReport) containing RSRP information and a candidate cell-list is transmitted by the UE and received at the s-eNB. The "A3 event" [7] is used as an entry condition to assess if the RSRP of the t-eNB is stronger than the RSRP of the s-eNB plus an hysteresis margin (called A3 offset). To trigger the MeasReport, the entry condition has to be valid during a specified time defined by the time to trigger (TTT) parameter [7]. Once the MeasReport is correctly received at the $\mathrm{s}-\mathrm{eNB}$, the HO preparation phase between t-eNB and s-eNB starts and a $\mathrm{HO}$ request is issued from the s-eNB to the t-eNB. The t-eNB then decides whether or not it can admit the UE and feedbacks this information to the s-eNB. Upon successful admission, the $\mathrm{HO}$ execution phase starts when the s-eNB transmits the $\mathrm{HO}$ command (HOcmd) to the UE with the necessary information to synchronize and perform initial access to the t-eNB. Upon successful reception of the HOcmd, the UE accesses the t-eNB, by means of a Random Access (RA) procedure via the RA Channel (RACH). With successful RA completion, the t-eNB receives a $\mathrm{HO}$ confirmation (HOconf) message from the UE. Finally, in the HO completion phase, the $\mathrm{s}-\mathrm{eNB}$ receives a $\mathrm{HO}$ complete message from the t-eNB that informs about the success of the HO process. Each of these phases contributes towards the overall signaling cost and latency to execute the HO. Hence, enhancing and optimizing these phases will facilitate the improvement of QoS and quality of experience (QoE) of the user/device. In order to achieve a good compromise between $\mathrm{HO}$ reliability and $\mathrm{HO}$ frequency, $\mathrm{HO}$ optimization deals, among others, with the adjustment of the TTT and A3 offset described above [8].

\section{System MOdEL}

A system-level LTE simulator is used considering a hexagonal grid deployment of 16 tri-sectored eNBs with cell wrap-around to allow fair interference conditions across the scenario. A set of 100 UEs is randomly placed over the scenario, where UEs follow a rectilinear motion at a fixed speed with initial random directions uniformly distributed between $\left[0^{\circ}\right.$, $360^{\circ}$ ]. A thorough description of the simulator's features and the considered HO model is covered in [5]. The main simulation assumptions are summarized in Table I.

Table I Simulation parameters and assumptions.

\begin{tabular}{|c|c|}
\hline Feature & Imple mentation \\
\hline Network topology & $\begin{array}{c}\text { A hexagonal grid of } 16 \times 3=48 \text { cells (wrap-around } \\
\text { included) }\end{array}$ \\
\hline Inter-site distance & From the set $\{100,200,400,600,800,1200\} \mathrm{m}$ \\
\hline System Bandwidth & $\begin{array}{c}B_{s y s}=5 \mathrm{MHz} \text { (paired FDD), with } N_{R B}^{D L}=N_{R B}^{U L}=25 \\
\text { RBs at carrier frequency } f_{c}=2.6 \mathrm{GHz}, 1 \mathrm{~TB}=6 \mathrm{RBs}, \\
\qquad N_{T B}^{D L}=N_{T B}^{U L}=\lfloor 25 / 6\rfloor\end{array}$ \\
\hline eNB DL power & $P_{e N B}=43 \mathrm{dBm}$ \\
\hline UE Power & $P_{U E}=23 \mathrm{dBm}$ \\
\hline Antenna patterns & 3D model specified in [9], Table A.2.1.1.2-2 \\
\hline Channel model & 6 tap model, Typical Urban (TU) \\
\hline Shadowing & $\begin{array}{c}\text { Log-normal shadowing: Mean } 0 \mathrm{~dB} \text {, Standard } \\
\text { deviation: } 8 \mathrm{~dB}\end{array}$ \\
\hline Propagation model & $L=130.5+37.6 \log _{10}(R), R$ in $\mathrm{km}$ \\
\hline UE speed & from the set $\{20,40,80,120\} \mathrm{km} / \mathrm{h}$ \\
\hline $\begin{array}{l}\text { RLF detection by L1 } \\
\text { of UE }\end{array}$ & $\begin{array}{r}\mathrm{T} 310=1 \mathrm{~s}, \mathrm{~N} 310=1, \mathrm{~N} 311=1 \text { as specified in }[10] \\
\mathrm{Q}_{\text {in }}=-4.8 \mathrm{~dB} ; \mathrm{Q}_{\text {out }}=-7.2 \mathrm{~dB} \text { as specified in }[11]\end{array}$ \\
\hline HO parameters & $\mathrm{TTT}=\{64,128,256\} \mathrm{ms}, \mathrm{A} 3$ offset $=\{2,4,5\} \mathrm{dB}$. \\
\hline
\end{tabular}

A. Received Power Consumption Model

In the eNB, the radio equipment can be basically divided into the Base-Band Unit (BBU) and the Remote Radio Unit (RRU). A BBU is responsible for communication through the physical interface. The BBU equipment is safely placed indoors and connected with RRU through optical fiber. An RRU is configured to communicate with the UE through the air interface. Similarly, for the UE, we will consider the contribution towards the received power consumption from the $\mathrm{RF}$ and BBU parts. A simplified overview of the eNB and UE components included in the received power model is shown in Fig. 2, where $P_{x, R x}$ is the received power, and $x=\{e N B, U E\}$. We are interested in the contribution of the $\mathrm{HO}$ mechanism towards supply power $P_{x, \text { sup }}$ necessary to retrieve the data.

In particular, we focus on the reception of $\mathrm{HO}$ signaling over the radio interface $(U u)$ in both DL and UL, namely: the MeasReport Rx, the RA preamble Rx, the HOconf Rx at eNB, and the HOcmd Rx at UE side (refer to Fig. 1). To derive the received power consumption of the above signaling reception, we proceed by deriving the size of such messages, then the amount of frequency resources needed to transmit these messages, and next the received power from these resources 
using a system-level simulator. Finally, the time duration of these messages is used to compute the time-averaged supply power consumption.

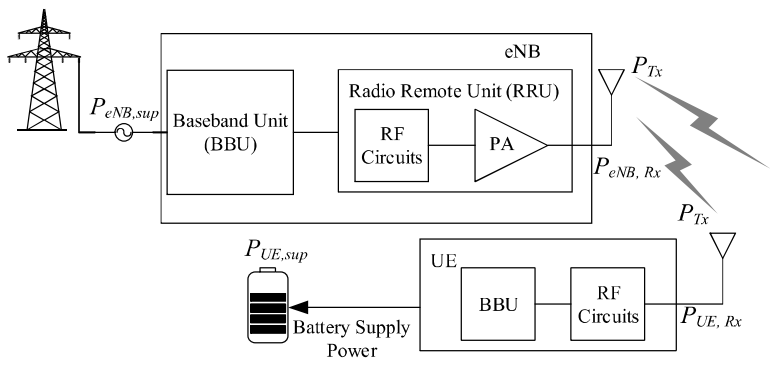

Fig. 2. A simplified overview of the eNB and UE components included in the received power model.

In LTE, the smallest time-frequency unit allocated to a UE is a resource block (RB). For a subcarrier spacing of $\Delta f=15$ $\mathrm{kHz}, \mathrm{a} \mathrm{RB}$ has a bandwidth of $B_{R B}=180 \mathrm{KHz}$ (i.e. $N_{S C}^{R B}=12$ subcarriers) and a time duration of one slot, $T_{R B}=T_{\text {slot }}=0.5$ $\mathrm{ms}$ [12]. The smallest resource unit consisting of one symbol and one subcarrier to which a modulated symbol is mapped onto is defined as a resource element (RE). According to the available modulation schemes in LTE, the number of carried bits in a single RE is $L_{R E} \in\{2,4,6\}$ bits for QPSK, 16-QAM, and 64QAM modulation respectively. A transport block is the amount of data that the upper layer (MAC layer) provides to the PHY layer depending on modulation and coding scheme (MCS) and cyclic redundancy check (CRC). Since HOs occur mostly at the cell-edge, where the RSRP is presumably low, this justifies our selection of QPSK as the modulation scheme used for the transmission of signaling messages (see Table II for details). To transmit MeasReport data bits in one TB, we assume $1 \mathrm{~ms}$ subframe with $6 \mathrm{RBs}$ and 2 slots. We also assume the modulation and coding scheme (MCS) index $I_{M C S}=0$ (very low) as a bottleneck for $\mathrm{HO}$ case scenario. Now we can find the number of information bits per TB ( $L_{T B}=152$ bits) using [13], which includes 128 bits for the MeasReport Rx and $24 \mathrm{CRC}$ bits.

Table II Power consumption parameters and values.

\begin{tabular}{|c|c|}
\hline Feature & Values \\
\hline $\begin{array}{l}\text { Signaling message } \\
\text { sizes }\end{array}$ & $\begin{array}{c}L_{M R}=128 \text { bits; } L_{H O c m d}=296 \text { bits; } L_{H O c n f}= \\
96 \text { bits (according to [14]) }\end{array}$ \\
\hline Carried bits in a TB & $\begin{array}{c}L_{T B}=152 \text { bits (with QPSK modulation, } L_{R E}=2, \\
\text { MCS index }=0 \text { ) [13] }\end{array}$ \\
\hline $\begin{array}{l}\text { Number of TBs per } \\
\text { each signaling } \\
\text { message }\end{array}$ & $\begin{array}{c}N_{T B}^{M R}=1 \mathrm{~TB} ; N_{T B}^{H O c n f}=1 \mathrm{~TB} ; N_{T B}^{H O c m d}=2 \\
\text { TBs; } N_{T B}^{R A}=1 \mathrm{~TB} .\end{array}$ \\
\hline $\begin{array}{c}\text { Power Amplifier } \\
\text { efficiency }\end{array}$ & $(31.1 \%)[2]$ \\
\hline RF supply power & $\begin{array}{c}P_{R F, e N B}=12.9 \mathrm{~W}[2] \text { and } P_{R F, U E}=2.35 \mathrm{~W}[4] \\
\text { scaled according to used bandwidth }\end{array}$ \\
\hline BBU power & $\begin{array}{c}P_{B B}^{\prime}=29.4 W[3], P_{T \times B B}=0.62 \mathrm{~mW}[4], \\
P_{R \times B B}=0.97 . R_{R x}+8.16(\mathrm{~mW})[4]\end{array}$ \\
\hline $\begin{array}{c}\text { Signaling } \\
\text { transmission times }\end{array}$ & $\begin{array}{c}T_{e N B}^{H O C m d}=1 \mathrm{~ms} ; T_{U E}^{M R}=1 \mathrm{~ms} ; T_{U E}^{H O c n f}=1 \mathrm{~ms} \\
T_{U E}^{R A C H t x}=1 \mathrm{~ms} \text { (we use preamble format } 0 \\
\text { according to the cell radius used in simulations, as } \\
\text { noted in [15]); }\end{array}$ \\
\hline
\end{tabular}

The size of each signaling message (in bits) is denoted by $L_{s}$, with subindex $s=\{M R, H O c m d, H O c n f\}$. With the above in mind the required number of TBs for each signaling message $s$, $N_{T B}^{S}$, can be obtained by:

$$
N_{T B}^{S}=\left\lceil\frac{L_{s}}{L_{T B}}\right\rceil, \text { with } s=\{M R, H O c m d, H O c n f\},
$$

where $\lceil x\rceil$ denotes the smallest integer larger than or equal to $x$, and, hence it is assumed herein that a signaling message requires an integer number of TBs. In addition, for the RACH signaling message transmission, since it carries an un-modulated preamble sequence, we can directly refer to the standard specifications which provide us with the value of $N_{T B}^{R A}$ [12] (see Table II).

For the case of an equal power allocation algorithm and according to the maximum available power at the eNB $\left(P_{e N B}\right)$ and at the UE $\left(P_{U E}\right)$ (see Table I), the allocated power per TB at the eNB and UE can be formulated as:

$$
\begin{gathered}
P_{e N B}^{T B}=P_{e N B} / N_{T B}^{D L}, \text { and } \\
P_{U E}^{T B}=P_{U E} / N_{T B}^{U L},
\end{gathered}
$$

where $N_{T B}^{D L}\left(N_{T B}^{U L}\right)$ is the total number of TBs in the DL (UL) given a system bandwidth $B_{\text {sys }}$ (see Table I).

The allocated transmitted power (in W) per signaling message $s$ is:

$$
P_{x, T x}^{s}=P_{x}^{T B} \cdot N_{T B}^{s}, \text { and }
$$

where $x=\{e N B, U E\}$ and, accordingly, the appropriate number of DL or UL signaling messages should be reflected in $N_{T B}^{S}$.

The received power (in $\mathrm{W}$ ) per signaling message $s$ is obtained using the system level simulator that follows the below expression,

$$
P_{x, R x}^{s}=P_{x, T x}^{s} \cdot G_{i, j} \cdot|h|^{2}+P_{n},
$$

where $x=\{e N B, U E\}, G_{i, j}$ is the total path gain for $i^{t h} \mathrm{UE}$ and $j^{\text {th }}$ t-eNB (including distance dependent attenuation, the angular antenna gain and shadow fading), $|h|^{2}$ is the fast fading contribution and, finally, $P_{n}$ is the additive white Gaussian noise contribution.

We apply well-known power consumption models for both the eNB and UE, [2][4], to obtain the received power necessary to receive the data for each of the signaling messages. In particular, the supply power for the eNB signaling $s$ reception, $P_{e N B, s u p}^{S, R x}$, can be written as

$P_{e N B, \text { sup }}^{s, R x}=P_{e N B, R x}^{S} / \eta+N_{T B}^{S} / N_{T B}^{D L} \cdot\left(P_{R F, e N B}+P_{B B}^{\prime}\right)$,

where $P_{e N B, R x}^{S}$ is the received power given by (5) and $\eta$ is the power amplifier efficiency. Equally, the supply power for the UE to receive signaling message $S, P_{U E, S u p}^{S, R x}$, is given by

$P_{U E, \text { sup }}^{s, R x}=P_{U E, R x}^{S}+N_{T B}^{S} / N_{T B}^{U L} \cdot\left(P_{R F, U E}+P_{R x B B}\left(R_{R x}\right)\right)$,

where $P_{U E, R x}^{S}$ is the received power given by (5), and where the received power contribution to the RF part is also scaled by the portion of utilized resources by signaling $S . P_{R x B B}$ is the received BBU power (see Table II) where $R_{R x}$ is the received data rate that can be found as $R_{x}^{S} \cdot L_{T B}[4]$. 

[6],

Now, the time-averaged supply power can be written as using

$$
\bar{P}_{x, \text { sup }}^{s, R x}=P_{x, \text { sup }}^{s, R x} \cdot T_{x}^{s} \cdot R_{x}^{s},
$$

where we have defined $R_{x}^{S}$ as the signaling rate which will be obtained from system level simulations.

\section{NUMERICAL RESULTS AND DISCUSSION}

In this section, we provide a numerical evaluation of the aggregate signaling rate and receiver power cost by $\mathrm{HO}$ signaling messages.

\section{A. Aggregate Signaling Rate Analysis}

The impact of ISD and UE speed on the signaling rate analysis during the $\mathrm{HO}$ procedure is shown in Fig. 3. One trend in the graph shows that increasing the UE speed increases the aggregate signaling rate. The other trend shows that both low and high ISDs have a higher aggregate signaling rate because of the UL impairments issue that we noted in our previous work [5]. For low ISDs, increased number of cell border crossing and high interference from the neighboring UEs result in increased $\mathrm{HO}$ rate and $\mathrm{HO}$ failure rate that further leads to an increased signaling rate. Similarly, for high ISDs, poor UL radio conditions for UEs close to cell borders cause the problem during $\mathrm{HO}$ procedure and thus have high signaling rate. Fig. 4 shows an average signaling rate distribution analysis for all simulated cases (a total 216 cases) of UE speed, ISD, TTT and offset values. The graph shows that the highest contribution to the airinterface signaling is the measurement report reception.

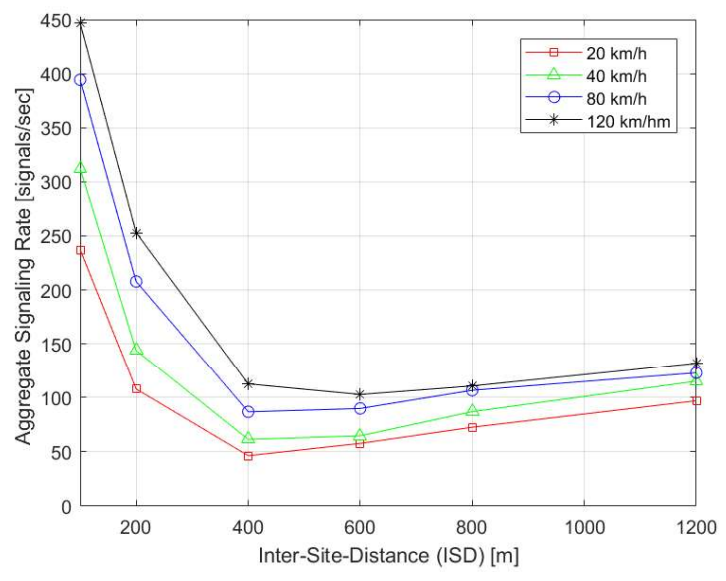

Fig. 3. Impact of ISD and UE speed on aggregate signaling rate, for fixed offset $=2 \mathrm{~dB}$ and TTT $=64 \mathrm{~ms}$.

The impact of varying the TTT values on the aggregate signaling rate is shown in Fig. 5. It is clear from the graph that increasing the TTT reduces the aggregate signaling rate significantly but at the cost of increased HO failures [8]. A similar trend is observed by varying the offset values. It is to be noted that low TTT and offset values have less HO failure cases, as early $\mathrm{HO}$ trigger prevents changing the radio link conditions [8].

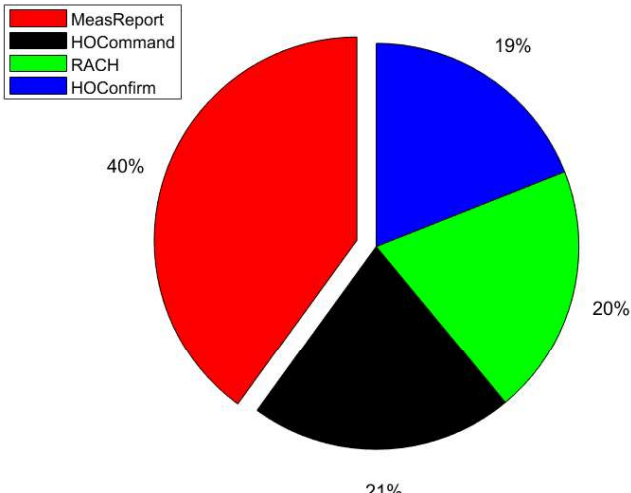

$21 \%$

Fig. 4. Average signaling rate distribution analysis for all simulated cases.

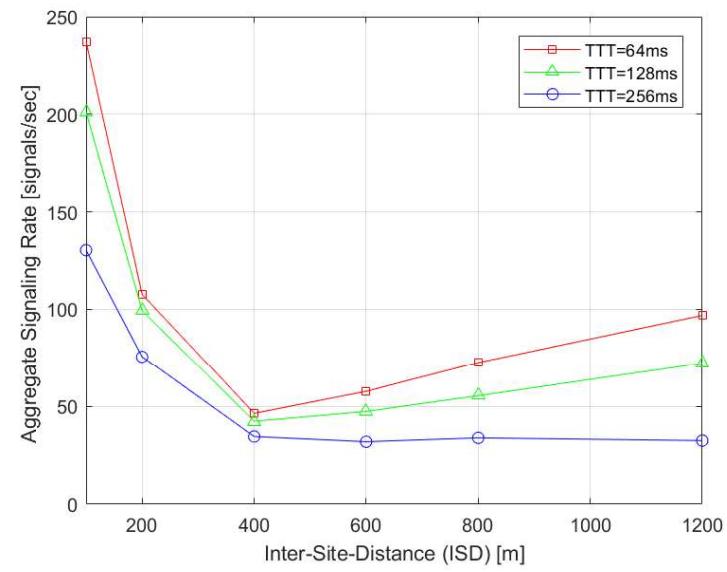

Fig. 5. Impact of varying TTT $\{64,128,256\} \mathrm{ms}$ on aggre gate signaling rate, for speed $=20 \mathrm{~km} / \mathrm{h}$ and $\mathrm{offset}=2 \mathrm{~dB}$.

\section{B. Receiver Power Consumption Analysis}

The impact of ISD and UE speed on the average supply power consumption of both the UE and the eNB, as given by (8), is shown in Fig. 6 and Fig. 7 respectively, at a constant TTT and A3 offset values. As expected, the power consumption of MeasReport, RACH, and Hoconf reception (eNB power consumption) is much higher compared to HOcmd reception (UE power consumption). It is clear from both graphs that the lowest ISD has the highest power consumption (due to high $\mathrm{HO}$ rate) then it decreases and, after that, it starts increasing again for high ISDs because of the UL impairments as also noted in [5]. We can say that ISD $=400 \mathrm{~m}$ presents a "sweet spot" ISD, out of the simulated cases, as we have the lowest receiver power consumption for this specific case. We can also observe that increasing UE speed, increases the power consumption especially at low ISDs, due to high aggregate signaling rate observed as noted in Fig. 3. 


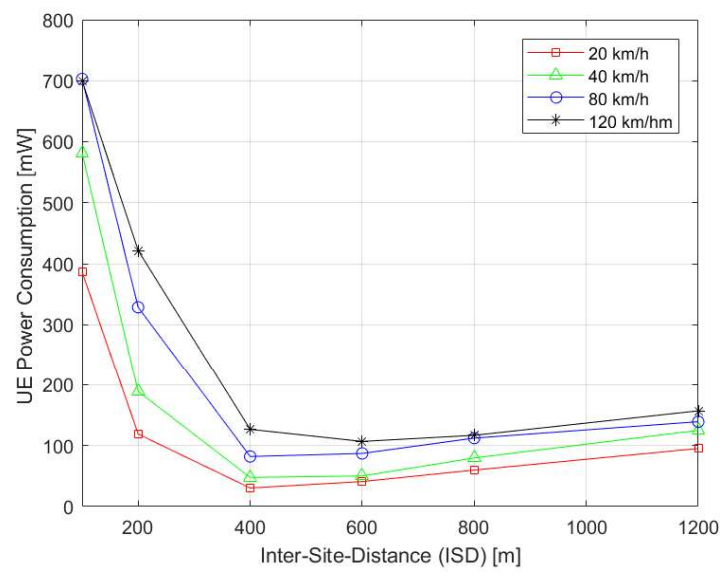

Fig. 6. Impact of ISD and UE speed on average supply power consumption of the UE received signaling messages, for offset $=2 \mathrm{~dB}$ and TTT $=64 \mathrm{~ms}$.

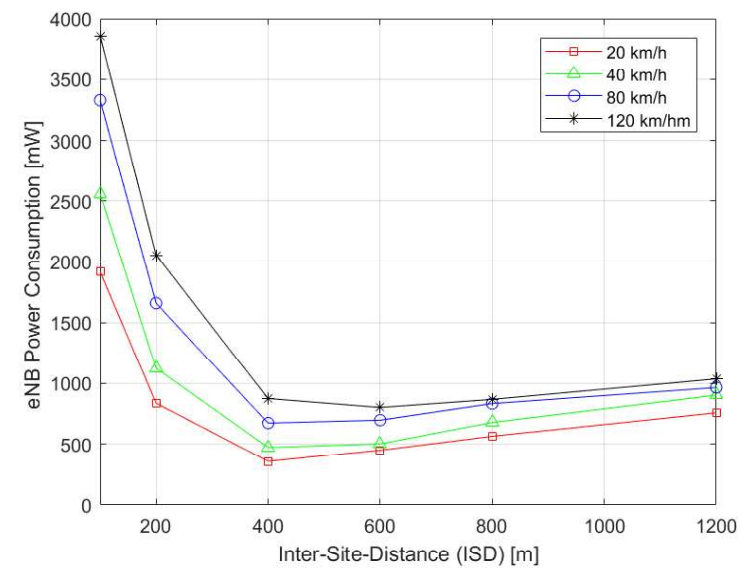

Fig. 7. Impact of ISD and UE speed on average supply power consumption of the $\mathrm{eNB}$ received signaling messages, for offset $=2 \mathrm{~dB}$ and $\mathrm{TTT}=64 \mathrm{~ms}$.

A per-type signaling power consumption breakdown for the eNB is shown in Fig. 8. The largest contributor to power consumption is the MeasReport reception by the eNB. This is because the UL transmission suffers from different channel impairments, due to transmission range and interference, for particular cell sizes. As a consequence, MeasReport retransmissions are often triggered which produces performance degradation in terms of increased power consumption.

Noting that increasing the A3 offset reduces the power consumption especially at low ISDs as shown in Fig. 9, however, this comes at the cost of increased HO failures as also noted in [8]. Similar behavior is observed by varying the TTT values.

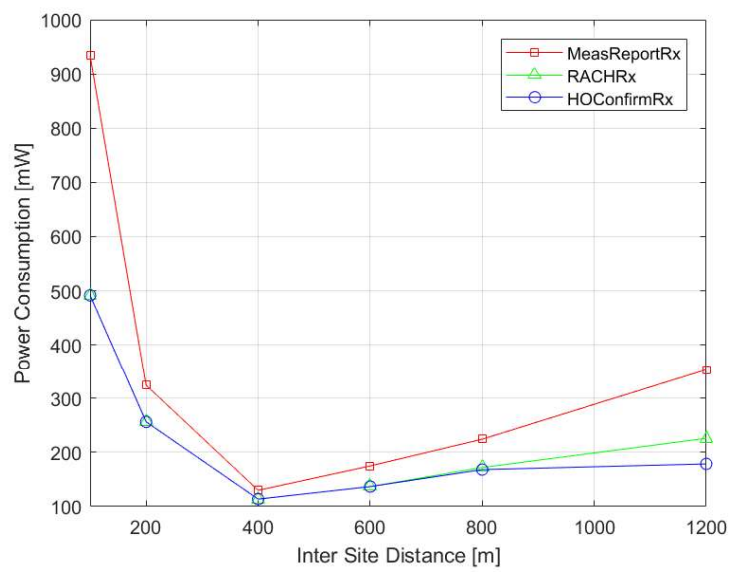

Fig. 8. Impact of ISD on average supply power consumption of various signaling messages reception at eNB for speed $=20 \mathrm{~km} / \mathrm{h}$, offset $=2 \mathrm{~dB}$ and TTT $=$ $64 \mathrm{~ms}$.

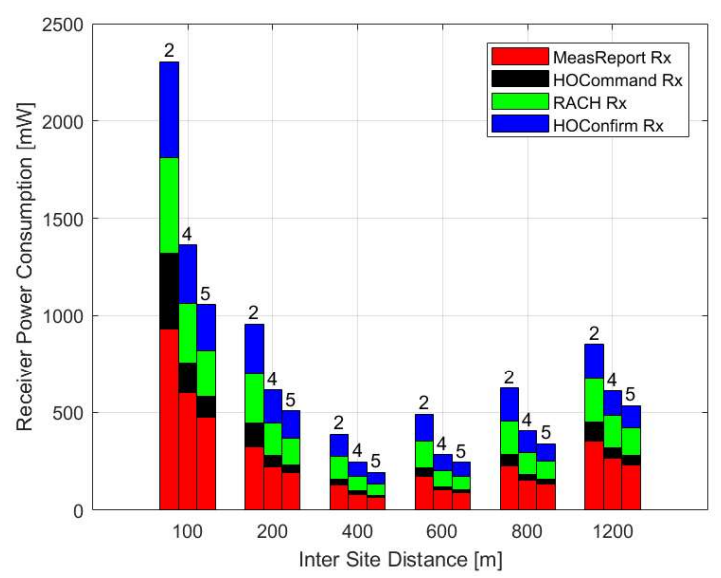

Fig. 9. Impact of ISD and offset $\{2,4,5\} \mathrm{dB}$ on average supply receiver power consumption, for speed $=20 \mathrm{~km} / \mathrm{h}$ and $\mathrm{TTT}=64 \mathrm{~ms}$.

\section{CONCLUSIONS}

In this paper, a simulation analysis is presented for the receiver power consumption during HO in an LTE network when various cell size, UE speed, offset and TTT values are applied. We observe that the largest contributor to the receiver power consumption is the reception of the measurement report by the eNB. We found a suitable cell size (ISD $=400 \mathrm{~m}$ out of the simulated cases) around which any increase or decrease of the cell size brings the performance degradation in terms of increased receiver power consumption. This work is in complete accordance with the uplink transmission limitations as noted in our previous work [5]. Also, increasing the offset and TTT values, the number of HOs decreases which result in reduction of the receiver power consumption, but at the cost of increased number of $\mathrm{HO}$ failures (as also noted in [8][5]). In future, we will propose a power efficient $\mathrm{HO}$ scheme to reduce the power consumption, especially by addressing the adequacy of the UL measurement report transmission. 


\section{REFERENCES}

[1] R. Arshad, H. Elsawy, S. Sorour, T. Y. Al-Naffouri and M. S. Alouini, "Handover Management in 5G and Beyond: A Topology Aware Skipping Approach", IEEE Access, vol. 4, pp. 9073-9081, Dec 2016.

[2] M. S. Mushtaq, S. Fowler, and A. Mellouk. "Power Saving Model for Mobile Device and Virtual Base Station in the 5G Era." IEEE International Conference on Communications (ICC), pp. 1-6, 2017.

[3] H. Holtkamp, et. al., "A Parameterized Base Station Power Model", IEEE Communications Letters, vol. 17, no. 11, pp. 2033-2035, Nov. 2013.

[4] M. Lauridsen et., "An Empirical LTE Smartphone Power Model with a View to Energy Efficiency Evolution" Inter technology Journal, vol. 18, pp. 172-193, 2014.

[5] M. Tayyab, G.P. Koudouridis, X. Gelabert "A Simulation Study on LTE Handover and the Impact of Cell Size". Broadband Communications, Networks, and Systems. (BROADNETS 2018).

[6] M. Tayyab, G. P. Koudouridis, X. Gelabert and R. Jäntti, "Signaling Overhead and Power Consumption during Handover in LTE", IEEE WCNC conference 2019. [Accepted]

[7] 3GPP TS 36.300 "(E-UTRA) and (E-UTRAN); Overall description; Stage 2 (Release 15)” V15.0.0, Section 10, pp. 93-143, Dec. 2017.
[8] X. Gelabert, G. Zhou and P. Legg, "Mobility Performance and Suitability of Macro Cell Power-Off in LTE Dense Small Cell HetNets", IEEE 18th International Workshop on Computer Aided Modeling and Design of Communication Links and Networks (CAMAD), pp. 99-103, 2013.

[9] 3GPP TR 36.814, "Further advancements for E-UTRA physical layer aspects (Release 9)," V9.0.0, Mar 2010.

[10] 3GPP TS 36.331, "E-UTRA Radio Resource Control (RRC); Protocol specification (Release 9)", v9.2.0, Mar 2010.

[11] 3GPP TS 36.133, "Requirements for support of radio resource management (Release 9)," v9.15.0, Mar 2013.

[12] 3GPP TS 36.211, "Physical channels and modulation (Release 15)," v15.1.0, Mar 2018

[13] 3GPP TS 36.213, "Evolved Universal Terrestrial Radio Access (EUTRA); Physical layer procedures", Table 7.1.7.2.1-1, Mar 2018.

[14] 3GPP R1-091791, "Handover performance in a Manhattan scenario," Huawei, May 2009.

[15] S. Sesia, I. Toufik, and M. Baker. "LTE--the UMTS Long TermEvolution: From Theory to Practice", (2nd Ed.) Wiley, 2011.

[16] J. Rodriguez et al., "SECRET - Secure Network Coding for Reduced Energy Next Generation Mobile Small cells", IEEE Internet Technologies and Applications (ITA) conference, pp. 329-333, 2017. 